\title{
QUINTUPLES WITH SQUARE TRIPLETS
}

\author{
STAN WAGON
}

\begin{abstract}
By applying modern computing power to an 1848 formula of C. Gill, we find five positive integers, every three of which sum to a square.
\end{abstract}

Diophantus $(\mathrm{V}, 17)$ gave a very simple method for getting four distinct positive integers such that the sum of every three is a perfect square (see [1, pp. 275-276] and for more background, also for the problem of integers with every pair summing to a square, see [3, problem D15]). For example, every triple from $\{1,22,41,58\}$ sums to a square. In 1848 , C. Gill [2, pp. 60-64] improved this by finding five distinct integers every three of which sum to a square. However, his methods did not guarantee positive integers; a typical example is: 639226, $684073,1384201,-660599,23401$.

Gill's method uses trigonometric functions to obtain a very complicated function $G(m / n, p / q)$ that churns out solutions (rational 5-tuples) for any pair of rational arguments. Of course, the rational output can just be scaled up to integers. Gill suggests that his formula ought to produce some positive solutions, "but the complexity of the formulas do not encourage the attempt". Modern software allows one, after replacing the trigonometric functions by their equivalent rational expressions, to easily implement a search for a positive solution, and a few minutes work with Mathematica showed that $G(23,11)$ is such (it yields the smallest example I have found; the search was based on integer arguments only). Thus, we obtain the following five integers near $10^{20}$, every triple from which sums to a square:

$$
\begin{array}{rrrrr}
26072 & 32331 & 15686 & 61931 \\
43744 & 83974 & 22825 & 91947 \\
1 & 18132 & 65441 & 36751 & 38222 \\
1 & 86378 & 73280 & 75870 & 76747 \\
5 & 19650 & 11481 & 49050 & 02347
\end{array}
$$

Although the computation is straightforward, Gill's formula- $G(m / n, p / q)-$ is quite massive when expressed purely in terms of $m / n$ and $p / q$. The interested reader can see the gigantic expression by programming the description below, using some high-precision symbolic software, and examining $v, w, x, y$, and $z$. The variable names (such as $s A B$ ) derive from Gill's use of trigono-

Received by the editor October 25, 1994.

1991 Mathematics Subject Classification. Primary 11E25, 11 D09.

(C)1995 American Mathematical Society 
metric functions (such as $\sin (A-B))$. Here is a description of $G$ :

$$
\begin{array}{ll}
\{c B, s B\}=\frac{\left\{p^{2}-q^{2}, 2 p q\right\}}{p^{2}+q^{2}} & \{c C, s C\}=\frac{\left\{m^{2}-n^{2}, 2 m n\right\}}{m^{2}+n^{2}} \\
s_{2}=2 s C c C & c_{2}=2 c C^{2}-1 \\
\alpha=1-s B s C+c B c_{2} & \beta=\frac{\alpha^{2}-(c B c C-c C)^{2}}{2 \alpha c B s C} \\
c A=1-2 /\left(1+\beta^{2}\right) & s A=(1-c A) \beta \\
s A B=s A c B-c A s B & c A B=s A s B+c A c B \\
a=(c C(c A+c B))^{2} & b=\left(s A B c C+c A s_{2}\right)^{2} \\
c=\left(s A B c C-c B s_{2}\right)^{2} & d=\left(c C-s B s_{2}+c C c A B\right)^{2} \\
e=\left(c C-s A s_{2}+c C c A B\right)^{2} & f=\left(c A+c B c_{2}+s C s A B\right)^{2} \\
g=\left(-c B-c A c_{2}+s C s A B\right)^{2} & x=w-b+d \\
v=(a+b+d-2 f) / 3 & w=v+f-d \\
y=x-a+b & z=(c+e+g-2 a) / 3
\end{array}
$$

Then $G(m / n, p / q)=\{v, w, x, y, z\}$ is a rational quintuple with square triplet sums (and $a-g$ are seven of the ten squares that arise in this way).

Using Mathematica's Reduce [] function, one discovers that the quintuplewith-square-triplets problem is equivalent (over the rationals or integers) to the quintuple-with-square-pairs problem, in the sense that one has a solution if and only if the other does; this was partially realized by Gill in 1848 . However, this equivalence does not preserve positiveness. So even though a positive solution to the quintuple-with-square-pairs problem has been found (Nicolas; see [3]), it does not directly yield a positive quintuple with square triplets.

\section{ACKNOWLEDGMENT}

I am grateful to the Reed College library for the loan of an original copy of [2].

\section{BIBLIOGRAPHY}

1. L. E. Dickson, History of the theory of numbers, Vol. 2, Chelsea, New York, 1971.

2. C. Gill, Application of the angular analysis to the solution of indeterminate problems of the second degree, Wiley, New York, 1848.

3. R. K. Guy, Unsolved problems in number theory, 2nd ed., Springer, New York, 1994.

Department of Mathematics, Macalester College, St. Paul, Minnesota 55105

E-mail address: wagon@macalstr.edu 